\title{
Radioprotective effects of induced astronaut torpor and advanced propulsion systems during deep space travel.
}

\author{
Squire, T 1,2 Ryan, A $3 \quad$ Bernard, S 4
}

\begin{abstract}
Affiliations
1. The Canberra Hospital, Department of Radiation Oncology. Garran. Australian Capital Territory. Australia

2. University of Notre Dame Australia, School of Medicine. Darlinghurst. New South Wales. Australia 3. University of Sydney. Applied and Plasma Physics Research Group. School of Aerospace Mechanical and Mechatronic Engineering. Camperdown. N.S.W. 2006. Australia

3. Université de Lyon. CNRS UMR5208 Institut Camille Jordan. Villeurbanne, France \& Inria Grenoble. France
\end{abstract}

\section{Keywords}

Circadian rhythm, cell cycle, torpor, radioprotection, galactic cosmic radiation, propulsion, solar particle event, bioastronautics

\section{Background}

Human metabolic suppression is not a new concept, with 1950s scientific literature and movies demonstrating its potential use for deep space travel (Hock.R, 1960). An artificially induced state of metabolic suppression in the form of torpor would improve the amount of supplies required and therefore lessen weight and fuel required for missions to Mars and beyond (Chouker.A et al, 2019). Transfer habitats for human stasis to Mars have been conceived (Bradford.J et al, 2018). Evidence suggests that animals, when hibernating, demonstrate relative radioprotection compared to their awake state. Experiments have also demonstrated relative radioprotection in conditions of hypothermia as well as during sleep (Bellesi.M et al, 2016 \& Andersen.M et al, 2009). Circadian rhythm disrupted cells also appear to be more susceptible to radiation damage compared to those that are under a rhythmic control (Dakup.P et al, 2018). 
An induced torpor state for astronauts on deep space missions may provide a biological radioprotective state due to a decreased metabolism and hypothermic conditions. A regular enforced circadian rhythm might further limit DNA damage from radiation. The As Low As Reasonably Achievable (A.L.A.R.A.) radiation protection concept defines time, distance and shielding as ways to decrease radiation exposure. Whilst distance cannot be altered in space and shielding either passively or actively may be beneficial, time of exposure may be drastically decreased with improved propulsion systems. Whilst chemical propulsion systems have superior thrust to other systems, they lack high changes in velocity and fuel efficiency which can be achieved with nuclear or electric based propulsion systems. Radiation toxicity could be limited by reduced transit times, combined with the radioprotective effects of enforced circadian rhythms during a state of torpor or hibernation.

\section{Objectives}

1. Investigate how the circadian clock and body temperature may contribute to radioprotection during human torpor on deep space missions.

2. Estimate radiation dose received by astronauts during a transit to Mars with varying propulsion systems.

\section{Methods}

We simulated three types of conditions to investigate the potential radioprotective effect of the circadian clock and decreased temperature on cells being exposed to radiation such that may be the case during astronaut torpor. These conditions were

- Circadian clock strength: strong vs weak.

- Light exposure: dark-dark vs light-dark cycle

- Body temperature: $37 \mathrm{C}$ vs hypothermia vs torpor.

We estimated transit times for a mission to Mars from Earth utilizing chemical, nuclear and electrical propulsion systems. Transit times were generated using the General Mission Analysis Tool (GMAT) and Matlab. These times were then input into the National Aeronautics and Space Administration (NASA) Online Tool for the Assessment of Radiation In Space (OLTARIS) computer simulator to estimate doses received by an astronaut for the three propulsion methods.

\section{Results}

Our simulation demonstrated an increase in radioprotection with decreasing temperature. The greatest degree of radioprotection was shown in cells that maintained a strong circadian clock during torpor. This was in contrast to 
relatively lower radioprotection in cells with a weak clock during normothermia. We were also able to demonstrate that if torpor weakened the circadian clock, a protective effect could be partially restored by an external drive such as lighting schedules to aid entrainment i.e: Blue light exposure for periods of awake and no light for rest times For the propulsion simulation, estimated transit times from Earth to Mars were 258 days for chemical propulsion with $165.9 \mathrm{mSv}$ received, 209 days for nuclear propulsion with $134.4 \mathrm{mSv}$ received and 80 days for electrical propulsion with $51.4 \mathrm{mSv}$ received.

\section{Conclusion}

A state of torpor for astronauts on deep space missions may not only improve weight, fuel and storage requirements but also provide a potential biological radiation protection strategy. Moreover, maintaining a controlled circadian rhythm during torpor conditions may aid radioprotection. In the not too distant future, propulsion techniques will be improved to limit transit time and hence decrease radiation dose to astronauts. Limiting exposure time and enhancing physiological radioprotection during transit could provide superior radioprotection benefits compared with active and passive radiation shielding strategies alone.

\section{Introduction}

Missions beyond the protection of the Earth's magnetosphere leave astronauts vulnerable to the harsh radiation environment in deep space. The constant stream of galactic cosmic radiation (GCR) and spontaneous solar particle events (SPE) from the Sun could lead to significant biological consequences including cardiovascular damage, neurocognitive impairment and carcinogenesis (Cucinotta.F, 2015). Whilst human data is lacking, a number of biological experiments suggest significant DNA damage occurs from cosmic radiation. Simulated high energy protons and Fe ions induced mutations distinct from gamma-rays and resulted in in-vivo development of T-cell acute lymphoblastic leukemia (Rodman.C et al, 2017). NASA's yearlong "Twins Study" investigated biological changes encountered during long human space flight. This study demonstrated a change in telomere length coupled with DNA methylation in immune cells, cardiovascular and cognitive effects (Garrett-Bakelman.F et al, 2019). Radiation mitigation strategies exist and could be largely grouped into modification of pre-launch, transit and habitation considerations (Squire.T et al, 2019). However, recent analysis of survival of astronauts and cosmonauts suggested that ionizing radiation exposure did not have a dramatic effect on risk of death due to cardiovascular

disease or cancer (Reynolds.R et al, 2019). Arguably, this data reflects missions within low Earth orbit and that biological effects beyond the magnetosphere may be much greater. 
A hypometabolic state is induced in animals during hibernation primarily to cope with a relative limited energy supply (Heldmaier.G et al, 2004). The idea of hibernation being a relative radioprotective state is not new with this being demonstrated experimentally in ground squirrels in the 1960s (Musacchia.X et al, 1968). It is hypothesized that during hibernation, radioprotection occurs via hypothermia (Lisowska.H et al, 2014) and decreased free radical induced DNA damage (Dang.B et al, 2014). Experiments have also demonstrated decreased radiation induced lethality of mice during an induced hypometabolic and hypothermic state. (Ghosh.S et al, 2017). Radioprotection during synthetic torpor has also been shown in irradiated rats (Tinganelli. W et al, 2019). Alterations in temperature are also known to affect the rate of change of biological or chemical systems. Hypothermia can be difficult to define but can be thought of a temperature below $35.5 \mathrm{C}$ in medical terms. Note should be made that hypothermia is a consequence of metabolic suppression during torpor. However, during an overwhelming exposure to cold, the human body increases in metabolic rate initially. A hypoxic tissue state may also limit permanency of DNA damage in the absence of oxygen. A meta-analysis of rodent experiments and mortality from published papers supported the hypothesis of hibernation increasing radioresistance (Cerri.M et al, 2016). From a design point of view, a smaller area of the spacecraft could be shielded from radiation during an induced torpor state for astronaut, which would reduce weight and improve launch costs and transit times.

The majority of biological functions are tightly regulated, with the mammalian cell cycle and the circadian clock operating in a rhythmic manner (El Cheikh.R et al, 2017 \& Matsuo.T et al, 2003). DNA damage responses, repair, apoptosis and checkpoint activity appear to be rhythmically controlled (Gaddameedhi.S et al, 2011, Bernard.S et al, 2006 \& Fu.L et al, 2003). Mice with a disrupted circadian clock experienced worse toxicity following irradiation compared with mice with a normal clock (Dakup.P et al, 2018). It appears circadian regulation is important in the heart for repair (Rabinovich-Nikitin.I et al, 2019), gut for microbiome regulation (Nobs.S et al, 2019 \& Godinho- Silva.C et al, 2019), bone formation regulation (Swanson.C et al, 2019) and central nervous system to maintain the neuroprotective effect of melatonin and limit oxidative stress in the context of Alzheimer's disease (Wu.H et al 2019). All of these organs are at specific risk from damage due to GCR (Boerma.M, 2016, Cucinotta.F et al, 2009, Datta.K et al, 2012, Hughson.R et al 2018; Kumar.S et al 2018) and therefore maintaining a circadian rhythm for astronauts may be important to maintain health and limit cellular damage from cosmic radiation (Astaburuaga.R. et al, 2019).

There is also evidence that a disrupted circadian rhythm can lead to colorectal cancer (Nunes, 2018), liver cancer (Kettner.N et al, 2016), lung cancer (Papagiannakopoulos.T et al, 2016) and oral cancers (Nirvani.M et al, 2018). In addition, sleep appears to be important to repair radiation induced DNA damage (Anderson.M et al, 2009 \& Bellesi.M et al, 2016). Melatonin may also play a role in radioprotection as higher levels during sleep may act as an antioxidant 
(Tordjman.S et al, 2017). During torpor, there may be a slowed stabilized cellular rhythm leading to relative radioprotective state particularly if feeding and lighting schedules are maintained. Total parenteral nutrition (TPN) may be utilized in the future for astronaut nutrition. Whilst TPN is currently administered intra-venously via a continuous 24-hour infusion to prevent metabolic instability, future administration regimens may alter the contents and rate to mimic a regular enteral feeding pattern.

Given the current evidence it may be beneficial to maintain a strong circadian rhythm during space flight. This would not only improve physiological effects from a normalized sleep wake cycle but also a potential radioprotective effect. This may be achievable during a state of torpor for astronauts on deep space missions.

Time of exposure to radiation could be reduced by utilizing more advanced propulsion systems to limit transit time in deep space. A significant proportion of the journey to another planet is unshielded between the Earth's magnetosphere to the atmospheric and planetary shielding provided by the destination planet. Chemical propulsion systems are the mainstay of current propulsion particular for manned spacecraft. Electrical propulsion systems which may utilize ion engines, have been in use since Deep Space 1 launched in 1998. Generally, these electrical systems are used to alter trajectory of satellites. An electrical propulsion system for a manned interplanetary mission would require a large amount of energy. Nuclear propulsion systems are a distinct possibility for future space travel.

This research aims to investigate which biological factors potentially contribute to radioprotection during human torpor on deep space missions as well as estimate radiation dose received by astronauts during a transit to Mars with chemical, nuclear and electrical propulsion systems.

\section{Methods}

Our hypothesis based on the current literature is that cell cycle progression could be slowed with a decrease in a temperature. Furthermore, cellular metabolism may be slowed along with cell division under hypothermic and torpor conditions. A strong clock under torpor conditions could provide an additional radioprotective benefit with decreased temperature during torpor (Figure.1)

\section{Three types of conditions were simulated in the model}

- Circadian clock strength: strong vs weak clock

- Light exposure: dark-dark vs light-dark cycle

- Body temperature: $37 \mathrm{C}$ vs hypothermia vs torpor 
In total, 20 runs were performed for each combination of condition $(2 \times 2 \times 3=$ 12 combinations) for a total of 240 simulations. In each simulation, around 60 dividing cells were simulated. Each cell was represented by two modules: one for the circadian clock and one for the cell cycle. The circadian clock was cell-autonomous and had a period around 24 hours. The clock drives the cell cycle by gating i.e.: preventing cell division at certain times of the day. We assumed that the cell cycle is in

part coordinated by the circadian clock and therefore timing of cell division and the rate at which cells divide is partly determined by circadian timing. This is in addition to the autonomous speed of progression in the cell cycle. It has been shown that the circadian clock can accelerate or slow down cell growth in a non-linear manner (El Cheikh et al, 2017). Cells were assigned a 1/100 probability of damage from radiation with damaged cells dividing faster than healthy cells.

The relative expression of two factors WEE1 and Mitosis Promoting Factor (MPF) orchestrated cell cycle progression along G1/S and G2/M phases in our simulation. WEE1 expression is assumed to be under control of the circadian clock transcription factor BMAL1. MPF is under weak control of the total cell number to reproduce cell contact inhibition. The timing of WEE1 and MPF was deterministic but division time was stochastically controlled by propensity function. At any time, cells could die with a propensity proportional to the total cell number in order to limit the number of cells that were simulated.

When a cell divides, the daughter cell inherits the characteristics of the mother cells with some variations. The clock period varies with a standard deviation of std_circadian_clock, and the cell becomes damaged with a probability mu_damage. A damaged cell has a cell cycle length target_period_damaged $25 \%$ smaller than the undamaged cell. Without the protective clock mechanism damaged cells proliferate advantageously along with a greater number of cells, this being a simplified model of neoplasia. At the end of the simulations, the ratio of undamaged healthy cells to total cell number provided a measure of how good the circadian clock was able to prevent the growth of damaged cells.

To simulate body temperature effects, we assumed that the target cell cycle lengths were correlated to the temperature with the cell cycle being slower at lower body temperatures. We changed a single temperature-dependent parameter to simulate different conditions between hypothermia and torpor. The autonomous cell cycle duration was set as $33 \%$ longer in torpor compared with hypothermia.

Temperature compensation is a defining feature of circadian clocks (Barrett. $\mathrm{R}$ et al, 1995). We assumed the circadian period is robust relative to the cell cycle length in response to changes in temperature. Although the circadian clock is likely to slow down with slower temperatures, we kept the period of the circadian clock constant in order to control for the generic radioprotective effect a slowing in metabolism would provide. Statistical analysis was performed using logistic regression on the ratio of healthy cells to normal cells. Further details in regards 
to default model parametres can be found in Supplementary data.

To investigate the effect of decreasing transit time to limit astronaut radiation exposure we used the General Mission Analysis Tool (GMAT) and Matlab simulations. To produce indicative and accurate simulation results, a series of assumptions were made regarding the specific propulsion systems used and the spacecraft itself. The chemical propulsion system was defined as a typical subcooled liquid oxygen propellant with an approximate vacuum specific impulse of 300s. The Nuclear system was a standard nuclear thermal propulsion device (NTP) with a specific impulse of $900 \mathrm{~s}$ and a peak thrust of $90 \mathrm{kN}$. The electric system was an X3 ion drive with a specific impulse of 4000s and a maximum thrust of $5.4 \mathrm{~N}$, to minimize duty cycle time a second X3 system was included for a total of two. The total spacecraft dry mass was set to 21 metric tonnes (Table 1).

For initial impulsive trajectory analysis, Hohmann and non-Hohmann style elliptic transfers were used comprising the Nuclear and Chemical propulsion system trajectories. This was performed using Matlab code written for the purposes of this paper, solving the equations of orbital motion in two dimensions. The Sun, Earth, and Mars ephemerides that were used for orbital characteristics and initial conditions were taken from datasets freely distributed by the NASA Jet Propulsion Laboratory. A three-dimensional analysis of an Earth Mars Hohmann transfer was performed using GMAT to successfully validate the accuracy of the two-dimensional Matlab code for Nuclear and Chemical propulsion. A key assumption of the trajectory design code was an initial Mars orbital transfer delta $\mathrm{V}$ of $3.56 \mathrm{~km} / \mathrm{s}$, initialized by a heavy lift launch vehicle. The low thrust electric Earth Mars trajectory was simulated and optimized using the ilt_opt Matlab script. This script adapts the Sparse Nonlinear Optimizer Software (SNOPT) to generate an iterative solution for minimum transfer time between Earth and Mars. This code solved the two-dimensional orbital motion equations, as with the other newly developed code.

Both Matlab codes produced a reasonable approximation of transfer time for each propulsion system, with the GMAT solution indicating minimal deviation from a two-dimensional formulation of Earth-Mars astrodynamics.

The transit times for chemical, nuclear and electrical propulsion were then input into the Online Tool for Assessment of Radiation in Space (OLTARIS) simulator which used HZETRN2005 and NUCFRG2 research codes for charge and energy transport. The environment type was set as free space galactic cosmic radiation using the Badhwar-O'Neill 2014 model. Timing of the transit was during a cycle of Solar Maximum during 2001 to take advantage of increased magnetic field shielding from the Sun. An aluminium/lithium alloy sphere was created around the computerized anatomical phantom to replicate the spacecraft hull to create a realistic simulation. Radiation dose in tissue and effective dose equivalent were calculated based on the modelled parameters. 


\section{Results}

Our modelling demonstrated that overall, radioprotection increased globally with decreasing temperature which was reflected in the ratio of healthy cells to total cell number. We observed that this ratio was greatest when a strong clock was maintained during torpor (0.9780518). This was in comparison of relatively lower radioprotection in conditions of weak clock control and normothermia (0.7118546). Although, lower radioprotection was observed with a strong clock compared with a weak clock in hypothermic conditions (Table 2).

An interesting observation was that a weak clock could be partially rescued by a light-day cycle. The significance of this finding is that if torpor weakened the internal circadian clock, the protective effect could be partially restored by an external drive such as light or feeding scheduling.

Without a protective clock mechanism, damaged cells have a proliferative advantage along with a greater number of cells - this being a simplified model of neoplasia. The radioprotective effect of a strong clock and torpor was demonstrated with the lowest total number of cells demonstrated under these conditions (40.8) particularly compared with the weak clock and normothermic condition (68.65). (Table 2)

At normothermic conditions the simulation demonstrated a higher ratio of healthy cells to total cells with strong clock control compared with weak clock control. (Table 2). This strong clock effect was most pronounced during normothermic conditions $(\mathrm{p}<2.2 \mathrm{e}-16)$ (Figure 2) and diminished during simulation under hypothermic $(\mathrm{p}=0.0007)$ (Figure 3$)$ and furthermore under torpor conditions $(\mathrm{p}=0.5128)$ (Figure 4).

Estimated transit times from Earth to Mars were 258 days for chemical (Figure 5), 209 days for nuclear (Figure 6) and 80 days for electrical propulsion (Figure 7). This resulted in astronaut effective doses of $165.9 \mathrm{mSv}, 134.4 \mathrm{mSv}$ and $51.4 \mathrm{mSv}$ respectively (Table 3). Using an electrical based propulsion system resulted in a $70 \%$ reduction in the effective dose received by the phantom compared to current chemical propulsion technology.

\section{Discussion}

\section{Hibernation, Torpor and circadian rhythm.}

Our simulation findings support our hypothesis that a strong circadian clock could provide an additional radioprotective benefit during a state of torpor.

Hibernation refers to specific species of animals that undergo long periods of decreased metabolic state with inter-bout episodes of arousal. Torpor is a state of metabolic suppression that may be induced artificially. Whilst human torpor is 
being investigated, targeted temperature management (TTM) with reduction in core temperature has been utilised in humans with neurological (Mattingly.T et al, 2019) as well as cardiac injury (Jauch.E et al, 2009). Cooling has been performed via high flow transnasal air in human volunteers (Nolan.J et al, 2019). Research by Schaffer et al suggest three main approaches to torpor states in humans. Body temperature based cooling, chemical/drug induction and brain/synaptic alteration (Schaffer. M et al, 2016). A step towards topor with induction of a shallow metabolic state may be achievable with acoustic and photoperiod stimulation as well as sedation with dexmedetomidine (Regan. M et al, 2020). For induction of torpor, development of pharmacologic agents is preferable compared to manipulation of the central nervous system by neurosurgical techniques (Cerri.M, 2017). Hypothermia alone cannot sufficiently create a safe synthetic torpor environment and knowledge must be gained from natural hibernators to orchestrate a favourable physiological environment (Nordeen. C et al, 2019)

A state of torpor may result in a relative tissue hypoxia which could facilitate radioprotection. However, a proportion of the galactic cosmic radiation are highly energetic charged particles which have a high linear energy transfer (LET). This would mean that the radiation could damage DNA directly and not rely on the presence of oxygen or free radicals to cause permanent damage.

There is mixed evidence as to what happens to the circadian rhythm in animals during hibernation or periods of torpor. What happens in humans is largely unknown. Experiments in hamsters have demonstrated a complete cessation of the circadian clock during hibernation (Revel. F et al, 2007). In grizzly bears, the circadian clock appears to continue unabated (Jansen. H et al, 2016). Denning female polar bears also appear to maintain circadian rhythm (Ware.J et al, 2020). In ground squirrels, circadian rhythm during torpor appears to be affected by seasonal changes (Williams.C et al, 2012). Therefore, in humans if the clock is shut down or slowed there may in fact be a loss of radioprotection and tumor suppression.

In order to maintain a circadian rhythm in humans during synthetic torpor perhaps it may be necessary to artificially create a light and dark environment utilizing controlled lighting with enough strength to penetrate the eyelids and stimulate the retina. For example, using blue light for periods of simulated daylight. Likely an intrinsic circadian rhythm exists however this may need to be reinforced with light exposure. Recent research suggests that there is significant interindividual variability in response of the human circadian rhythm to light and melatonin levels may be significantly suppressed even with low levels of light exposure altering circadian rhythm which may impact health (Phillips. A. et al, 2019). Although, the effect of age and sex was not specified in the study. Regular feeding schedules may also be beneficial which may be facilitated via percutaneous feeding tubes given the astronaut torpor state.

Other research suggests that there are inherent sex differences in circadian timing systems including the hypothalamic-pituitary-gonadal axis, hypothalamicadrenal-pituitary axis and sleep-arousal systems (Bailey et al, 2014). This may 
have implications on astronaut gender for induced hibernation. Studies in older mice indicate an age related circadian disorganization with peripheral circadian clocks being entrained with meal timing (Tahara.Y et al, 2017).

A complicating factor of biological response to cosmic radiation exposure is that there may be cell cycle phase advancement following radiation induced DNA damage with this effect being demonstrated in vitro and in vivo experiments. This may effectively "reset" the circadian clock. (Oklejewicz.M et al, 2008)

Aside from potential physiological advantages, torpor chambers also present a beneficial structural alteration whereby a smaller area can be shielded within the spacecraft. Perhaps, artificial intelligence may be able to navigate the ship unshielded with human astronauts protected in chambers with shielding utilizing optimal nanomaterials.

\section{Circadian rhythm during planetary habitation}

Alteration in circadian rhythm may not only occur during torpor but also during habitation on planets such as Mars. Due to the dusty atmosphere humans may not receive circadian signals such as blue light to stimulate the retina and facilitating an awake state. There may also be loss of optimal sunlight during habitation on Mars if humans will live underground to facilitate radiation shielding using Martian regolith.

Although a Martian day is only marginally longer than a day experienced on Earth. In the future, colonizing a moon around Saturn such as Titan would result in the inhabitant experiencing a time of 15 days to orbit Saturn. If interstellar travel is feasible in the future, Kepler-186f located 490 light- years from Earth may be habitable. This planet takes about 130 days to orbit its red dwarf star. As a result, the future human inhabitants of other planets would likely experience alteration in circadian rhythm. In addition to altered circadian rhythm alone there may be synergistic effects of microgravity, psychological stress and lack of sleep leading to increased DNA damage from radiation (Squire.T et al, 2019)

\section{Propulsion systems}

Our results demonstrate the shortest transit time and therefore the lowest radiation dose received by astronauts when comparing systems was utilizing electrical propulsion. This is due to the system's ability to be in near constant operation throughout the mission, speeding up more than is possible with other impulsive rockets. This efficiency is mathematically described by specific impulse, or the time a thruster can maintain an acceleration of $9.81 \mathrm{~m} / \mathrm{s} / \mathrm{s}$ with a unit of fuel. The nuclear system used in this study has a specific impulse three times that of the chemical system, and the electric system is over 12 times greater than the chemical system. It is also important to consider the relative system weights, as a nuclear system can add a significant mass to a spacecraft, reducing 
delta V. Furthermore, for a low thrust device such as an electric thruster, the thrust is an important factor. If the thrust is too low the rocket will take longer than the mission timeframe to use up all the fuel to achieve a maximum speed and then slow down.

The two X3 ion thrusters are capable of achieving a high enough thrust for an Earth Mars mission. Whilst this is an attractive concept, electric propulsion systems are now currently used to shift directions of smaller satellites. For a manned mission to Mars an electrical system would require an immense amount of energy in excess of $1 \mathrm{MW}$ during our simulation. Nonetheless, significant technological advances are currently being made paving the way for faster interplanetary travel. Policy regarding the use of technologically available nuclear thermal generators in space for power generation and propulsion is beginning to show signs of progress after decades of hiatus. This is a good sign that the power required to propel large spacecraft with electric propulsion may be available in the next decade.

\section{Conclusion}

A state of induced torpor for astronauts may provide a radioprotective strategy in the future. This would provide an additional benefit to improved weight and therefore fuel requirements for deep space travel. Our simulation suggests an enforced circadian rhythm could further improve radioprotection and may play a significant role in the cellular response to radiation induced DNA damage. An enforced rhythm could be facilitated by lighting and feeding schedules. Our propulsion simulation demonstrated a decreased effective radiation dose received by an astronaut by $70 \%$ when an electrical propulsion system was used compared with current day chemical propulsion.

\section{References}

Andersen ML, Ribeiro DA, Bergamaschi CT, Alvarenga TA, Silva A, Zager A, Campos RR, Tufik S. Distinct effects of acute and chronic sleep loss on DNA damage in rats. Progress in Neuro- Psychopharmacology and Biological Psychiatry. 2009 Apr 30;33(3):562-7.

Astaburuaga R, Basti A, Li Y, Herms D, Relégio A. Circadian regulation of physiology: Relevance for space medicine. REACH. 2019 Jun 1;14:100029.

Bailey M, Silver R. Sex differences in circadian timing systems: implications for disease. Frontiers in neuroendocrinology. 2014 Jan 1;35(1):111-39.

Barrett RK, Takahashi JS. Temperature compensation and temperature entrainment of the chick pineal cell circadian clock. Journal of Neuroscience. 1995 Aug 
1;15(8):5681-92.

Bellesi M, Bushey D, Chini M, Tononi G, Cirelli C. Contribution of sleep to the repair of neuronal DNA double-strand breaks: evidence from flies and mice. Scientific reports. 2016 Nov 10;6:36804.

Bernard S, Herzel H. Why do cells cycle with a 24-hour period? Genome Informatics. 2006;17(1):72- 9 .

Boerma. M. An introduction to space radiation and its effects on the cardiovascular system. NASA. 2016.

Bradford J, Schaffer M, Talk D. Torpor inducing transfer habitat for human stasis to Mars. NASA. 2018.

Cerri M, Tinganelli W, Negrini M, Helm A, Scifoni E, Tommasino F, Sioli M, Zoccoli A, Durante M. Hibernation for space travel: Impact on radioprotection. Life sciences in space research. 2016 Nov 1;11:1-9.

Cerri M. The central control of energy expenditure: exploiting torpor for medical applications. Annual review of physiology. 2017 Feb 10;79:167-86.

Choukèr A, Bereiter-Hahn J, Singer D, Heldmaier G. Hibernating astronautsscience or fiction? Pflügers Archiv-European Journal of Physiology. 2019 Jun $1 ; 471(6): 819-28$.

Cucinotta FA. Review of NASA approach to space radiation risk assessments for Mars exploration. Health physics. 2015 Feb 1;108(2):131-42.

Cucinotta FA, Wang H, Huff JL. Risk of acute or late central nervous system effects from radiation exposure. Human health and performance risks of space exploration missions: evidence reviewed by the NASA Human Research Program. 2009;191:212.

Dakup PP, Porter KI, Cheng Z, Gaddameedhi S. Circadian clock protects against radiation-induced dermatitis and cardiomyopathy in mice. AACR. 2018.

Dang B, Yang Y, Zhang E, Li W, Mi X, Meng Y, Yan S, Wang Z, Wei W, Shao C, Xing R. Simulated microgravity increases heavy ion radiation-induced apoptosis in human B lymphoblasts. Life sciences. 2014 Mar 3;97(2):123-8.

Datta K, Suman S, Kallakury BV, Fornace Jr AJ. Exposure to heavy ion radiation induces persistent oxidative stress in mouse intestine. PloS one. 2012 Aug 24;7(8):e42224.

El Cheikh R, Bernard S, El Khatib N. A multiscale modelling approach for the regulation of the cell cycle by the circadian clock. Journal of theoretical biology. 2017 Aug 7;426:117-25.

Fu L, Lee CC. The circadian clock: pacemaker and tumour suppressor. Nature Reviews Cancer. 2003 May;3(5):350. 
Gaddameedhi S, Selby CP, Kaufmann WK, Smart RC, Sancar A. Control of skin cancer by the circadian rhythm. Proceedings of the National Academy of Sciences. 2011 Nov 15;108(46):18790-5.

Garrett-Bakelman FE, Darshi M, Green SJ, Gur RC, Lin L, Macias BR, McKenna MJ, Meydan C, Mishra T, Nasrini J, Piening BD. The NASA Twins Study: A multidimensional analysis of a year-long human spaceflight. Science. 2019 Apr 12;364(6436):eaau8650.

Ghosh S, Indracanti N, Joshi J, Ray J, Indraganti PK. Pharmacologically induced reversible hypometabolic state mitigates radiation induced lethality in mice. Scientific reports. 2017 Nov 2;7(1):1-4.

Godinho-Silva C, Domingues RG, Rendas M, Raposo B, Ribeiro H, da Silva JA, Vieira A, Costa RM, Barbosa-Morais NL, Carvalho T, Veiga-Fernandes H. Light-entrained and brain-tuned circadian circuits regulate ILC3s and gut homeostasis. Nature. 2019 Sep 18:1-5.

Heldmaier G, Ortmann S, Elvert R. Natural hypometabolism during hibernation and daily torpor in mammals. Respiratory physiology \& neurobiology. 2004 Aug $12 ; 141(3): 317-29$.

Hock RJ. The potential application of hibernation to space travel. Aerosp. Med. 1960 Jun;31:485-9.

Hughson RL, Helm A, Durante M. Heart in space: effect of the extraterrestrial environment on the cardiovascular system. Nature Reviews Cardiology. 2018 Mar;15(3):167.

Jansen HT, Leise T, Stenhouse G, Pigeon K, Kasworm W, Teisberg J, Radandt T, Dallmann R, Brown S, Robbins CT. The bear circadian clock doesn't 'sleep'during winter dormancy. Frontiers in zoology. 2016 Dec;13(1):42.

Jauch EC, Kern KB. Post-cardiac arrest syndrome: epidemiology, pathophysiology, treatment, and prognostication: a scientific statement from the International Liaison Committee on Resuscitation; the American Heart Association Emergency Cardiovascular Care Committee; the Council on Cardiovascular Surgery and Anesthesia; the Council on Cardiopulmonary, Perioperative, and Critical Care; the Council on Clinical Cardiology; the Council on Stroke (part 1). International emergency nursing. 2009 Oct 1;17(4):203-25.

Kettner NM, Voicu H, Finegold MJ, Coarfa C, Sreekumar A, Putluri N, Katchy CA, Lee C, Moore DD, Fu L. Circadian homeostasis of liver metabolism suppresses hepatocarcinogenesis. Cancer cell. 2016

Kumar S, Suman S, Fornace AJ, Datta K. Space radiation triggers persistent stress response, increases senescent signaling, and decreases cell migration in mouse intestine. Proceedings of the National Academy of Sciences. 2018 Oct 16;115(42):E9832-41.

Lisowska H, Brehwens K, Zülzer F, Wegierek-Ciuk A, Czub J, Lankoff A, 
Haghdoost S, Wojcik A. Effect of hypothermia on radiation-induced micronuclei and delay of cell cycle progression in TK6 cells. International journal of radiation biology. 2014 Apr 1;90(4):318-24.

Matsuo T, Yamaguchi S, Mitsui S, Emi A, Shimoda F, Okamura H. Control mechanism of the circadian clock for timing of cell division in vivo. Science. 2003 Oct 10;302(5643):255-9.

Mattingly TK, Lownie SP. Cold blood perfusion for selective hypothermia in acute ischemic stroke. Brain Circulation. 2019 Oct 1;5(4):187.

Meholic. G. Advanced Space Propulsion Concepts for Interstellar Travel. American Institute of Aeronautics and Astronautics (AIAA). 2008 Musacchia XJ, Barr RE. Survival of whole-body-irradiated hibernating and active ground squirrels; Citellus tridecemlineatus. Radiation research. 1968 Feb 1;33(2):348-56.

Nirvani M, Khuu C, Utheim TP, Sand LP, Sehic A. Circadian clock and oral cancer. Molecular and clinical oncology. 2018 Feb 1;8(2):219-26. Nobs SP, Tuganbaev T, Elinav E. Microbiome diurnal rhythmicity and its impact on host physiology and disease risk. EMBO reports. 2019 Apr 1;20(4).

Nolan JP, Neumar RW, Adrie C, Aibiki M, Berg RA, Bbttiger BW, Callaway C, Clark RS, Geocadin RG, Chava R, Zviman M, Assis FR, Raghavan MS, Halperin H, Maqbool F, Geocadin R, Quinones-Hinojosa A, Kolandaivelu A, Rosen BA, Tandri H. Effect of high flow transnasal dry air on core body temperature in intubated human subjects. Resuscitation. 2019 Jan 1;134:49-54.

Nordeen CA, Martin SL. Engineering human stasis for long-duration spaceflight. Physiology. 2019 Mar 1;34(2):101-11.

Nunes, M. (2018). Disruption of Circadian Rhythm and its Impact on the Progression of Colorectal Cancer in the Murine Animal Model.

Oklejewicz M, Destici E, Tamanini F, Hut RA, Janssens R, van der Horst GT. Phase resetting of the mammalian circadian clock by DNA damage. Current Biology. 2008 Feb 26;18(4):286-91.

Papagiannakopoulos T, Bauer MR, Davidson SM, Heimann M, Subbaraj L, Bhutkar A, Bartlebaugh J, Vander Heiden MG, Jacks T. Circadian rhythm disruption promotes lung tumorigenesis. Cell metabolism. 2016 Aug 9;24(2):32431.

Phillips AJ, Vidafar P, Burns AC, McGlashan EM, Anderson C, Rajaratnam SM, Lockley SW, Cain SW. High sensitivity and interindividual variability in the response of the human circadian system to evening light. Proceedings of the National Academy of Sciences. 2019 Jun 11;116(24):12019-24.

Rabinovich-Nikitin I, Lieberman B, Martino TA, Kirshenbaum LA. Circadianregulated cell death in cardiovascular diseases. Circulation. 2019 Feb 12;139(7):965-80. 
Regan MD, Flynn-Evans EE, Griko YV, Kilduff TS, Rittenberger JC, Ruskin KJ, Buck CL. Shallow metabolic depression and human spaceflight: a feasible first step. Journal of Applied Physiology. 2020 Mar 1;128(3):637-47.

Revel FG, Herwig A, Garidou ML, Dardente H, Menet JS, Masson-Pévet M, Simonneaux V, Saboureau M, Pévet P. The circadian clock stops ticking during deep hibernation in the European hamster. Proceedings of the National Academy of Sciences. 2007 Aug 21;104(34):13816-20.

Reynolds RJ, Bukhtiyarov IV, Tikhonova GI, Day SM, Ushakov IB, Gorchakova TY. Contrapositive logic suggests space radiation not having a strong impact on mortality of US astronauts and Soviet and Russian cosmonauts. Scientific reports. 2019 Jul 4;9(1):8583.

Rodman C, Almeida-Porada G, George SK, Moon J, Soker S, Pardee T, Beaty M, Guida P, Sajuthi SP, Langefeld CD, Walker SJ. In vitro and in vivo assessment of direct effects of simulated solar and galactic cosmic radiation on human hematopoietic stem/progenitor cells. Leukemia. 2017 Jun;31(6):1398.

Schaffer M, Bradford J, Talk D. A feasible, near-term approach to human stasis for long-duration deep space missions. In67th International Astronautical Congress (IAC), Guadalajara, Mexico 2016 Sep 26 (pp. 1-17).

Squire T, Buchanan G, Elsaleh H. Mission to Mars: Radiation Safety or Radiation Disaster? Space Transit and Mars Radiation Exposure Risks-The Shielding Effect of a Graphene Space Suit and a Storm Shelter During Space Travel. Journal of the Australasian Society of Aerospace Medicine. Vol 11. 2019 (pp.1-9)

Swanson CM, Kohrt WM, Wolfe P, Wright KP, Shea SA, Cain SW, Munch M, Vujovié N, Czeisler CA, Orwoll ES, Buxton OM. Rapid suppression of bone formation marker in response to sleep restriction and circadian disruption in men. Osteoporosis International. 2019 Aug 24:1-9.

Tahara Y, Takatsu Y, Shiraishi T, Kikuchi Y, Yamazaki M, Motohashi H, Muto A, Sasaki H, Haraguchi A, Kuriki D, Nakamura TJ. Age-related circadian disorganization caused by sympathetic dysfunction in peripheral clock regulation. npj Aging and Mechanisms of Disease. 2017 Jan 5;3(1):1-1.

Tinganelli W, Hitrec T, Romani F, Simoniello P, Squarcio F, Stanzani A, Piscitiello E, Marchesano V, Luppi M, Sioli M, Helm A. Hibernation and Radioprotection: Gene Expression in the Liver and Testicle of Rats Irradiated under Synthetic Torpor. International journal of molecular sciences. 2019 Jan;20(2):352.

Tordjman S, Chokron S, Delorme R, Charrier A, Bellissant E, Jaafari N, Fougerou C. Melatonin: pharmacology, functions and therapeutic benefits. Current neuropharmacology. 2017 Apr 1;15(3):434-43.

Ware JV, Rode KD, Robbins CT, Leise T, Weil CR, Jansen HT. The Clock Keeps Ticking: Circadian Rhythms of Free-Ranging Polar Bears. Journal of Biological Rhythms. 2020 Apr;35(2):180-94. 
Williams CT, Barnes BM, Buck CL. Daily body temperature rhythms persist under the midnight sun but are absent during hibernation in free-living arctic ground squirrels. Biology letters. 2012 Feb 23;8(1):31-4.

Wu H, Dunnett S, Ho YS, Chang RC. The role of sleep deprivation and circadian rhythm disruption as risk factors of Alzheimer's disease. Frontiers in neuroendocrinology. 2019 May 15:100764. 